\title{
Energy-based RRH assignment scheme for Heterogeneous Cloud Radio Access Networks
}

\author{
Raymond Gbegbe \\ INPHB, Yamoussoukro \\ Cote d'Ivoire
}

\author{
Soumaya Hamouda \\ MEDIATRON Lab., Sup'Com \\ Tunisia
}

\author{
Guy Landry Diety \\ INPHB, Yamoussoukro \\ Côte d'Ivoire
}

\begin{abstract}
One of the main concerns of the next fifth mobile radio generation $(5 \mathrm{G})$ is increasing the network radio capacity while reducing system power consumption. Cloud-Radio Access Network (CRAN) coupled with Heterogeneous Networks (HetNets), called H-CRAN, reveals a promising paradigm to solve this problem. By having one centralized Base Band Unit (BBU) pool, the transmit powers at the different Radio Remote Headers (RRHs) are indeed better controlled while meeting users' quality of service. More precisely, a coordinated power control at the H-CRAN's BBU pool helps activating the appropriate RRHs in the macrocell given the fact that coverage and radio capacity are ensured with the HetNet. In this paper, a practical solution to efficiently monitor the transmit power at the RHHs in an $\mathrm{H}_{-}$ CRAN is proposed. Only the most requested RRHs and which ensure minimum total power consumption in the system are activated. Different scenarios are studied and simulation results showed that the total transmit power can be reduced by at least $58 \%$.
\end{abstract}

\section{General Terms}

5G, CRAN, System architecture.

\section{Keywords}

CRAN, H-CRAN, RRH, Power control, Energy Efficiency

\section{INTRODUCTION}

To cope with the explosion of multimedia traffic demands in terms of data rates and the number of connected objects, current and future networks must face major technical challenges in order to increase their radio capacity. Besides, operations pay more attention to the Capital Expenditure (CAPEX) and Operating Expenses (OPEX) nowadays. Numerous studies have shown that Heterogeneous Networks (HetNets) networks and Cloud Radio Access Network (CRAN) are promising solutions to these challenges and above all they improve the Spectral Efficiency (SE) as well as the Energy Efficiency (EE) of the systems [1,4]. The principle of the HetNets consists in integrating Low Power Nodes (LPNs) or small cells of micro, pico or femto types inside macrocells served by Macro-Base Stations (MBS) or High Power Node (HPN) $[2,3,11]$. This increases the capacity and coverage of the network because the frequency reuse distance is reduced. On the other hand, C-RAN is a centralized architecture in which the base station processing part of the different Base Stations (BSs) is separated from the radiofrequency processing subsystem [4]. The baseband processing is carried out by Base Band Units (BBUs) which are grouped together on a centralized site called BBU pool, whereas Radiofrequency processing is achieved at the Remote Radio Headers (RRHs). H-CRAN (Heteregeneous Cloud Access Network) is a new architecture resulting of coupling C-RAN with HetNets. All BBUs of both macrocells and the corresponding small cells are gathered in one single BBU pool. The H-CRAN is considered by experts as a promising solution to meet the demand for broadband and energy reduction as advocated by $5 \mathrm{G}$ networks [2]. Through optimal BBU management, it can indeed better coordinate the transmit power in the network, mitigate the interference, increase the radio capacity and reduce system energy consumption while ensuring a total coverage in the network and meet users' quality of service requirements.

Recent research has identified mechanisms to optimize the SE and the EE in C-RANs. For example, some contributions have examined the clustering techniques to determine the best BBU-RRH association in order to minimize the number of RRHs allocated to a data stream without degrading the required QoS [5,7]. Other proposed clustering methods aim at maximizing the number of RRHs by BBU in [7]. Neighboring RRHs are assigned to the same BBU as long as the processing capacity limit is not reached $[5,6]$. By reducing the number of active BBUs or RRHs, all of these clustering mechanisms help reduce the power consumption in the system. In addition, other studies have focused on methods for putting the BBUs or RRHs on standby to increase the EE [12]. In [8], the authors proposed a joint mechanism for RRH activation and the radio resource allocation to maximize the $\mathrm{EE}$ in a $\mathrm{H}$ CRAN, under the constraint of the fronthaul link capacity. The authors of $[9,10]$ studied the mechanisms which minimizes both the total power consumed and the number of failures of standby/ active modes in a H-CRAN network with guaranteed QoS.

In this paper, a new algorithm which minimizes the total power consumed by the H-CRAN under quality of users' constraint is proposed. The basic idea is to identify the RRH which are underutilized, then switch them to standby mode and keep on the RRH which are the most requested and whose transmit power is higher than a certain threshold. The User Equipments (UEs) associated to the first RRHs' group, will then be reassigned to the RRHs which are not overloaded while respecting their quality of service and minimizing the total consumed power in the network. The threshold is indeed set such that the total consumed power is minimized while the user's throughput is reached.

The paper is organized as follows. Section 2 describes the system model and formulates the optimization problem. Section 3 gives more details about the proposed algorithm. Simulation results are presented and commented in Section 4. Finally, Section 5 concludes this paper. 


\section{SYSTEM MODEL AND PROBLEM FORMULATION}

\subsection{System layout}

The considered system consists of an H-CRAN network architecture including a set of virtual BBUs grouped in a BBU pool connected to one macrocell and several small cells inside, and the corresponding RRHs which are responsible for the radio transmission (Fig. 1). The RRHs are randomly distributed inside the macrocell. It is also assumed that the end users can receive data from either the RRH of the small cells or the RRH of the macrocell (M-RRH) since the small cells are considered as working in open access mode. In addition, the BBU Pool is connected to all the base stations by a high-speed fiber optic link.

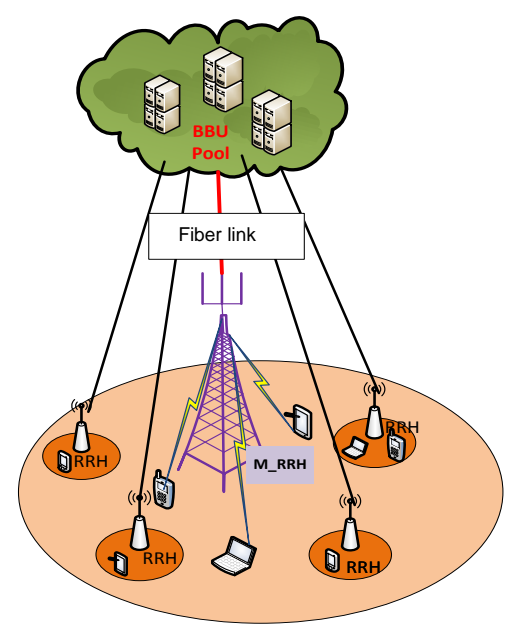

Fig. 1: System layout

\subsection{Energy consumption model and problem formulation}

Since the study's aim is minimizing the total consumed power at the RRH by properly activating the RRH and reassigning the UEs to these RRHs, here below is given the expression of the total consumed power at the fronthaul without considering the the Fiber Optic link power consumption.

Let $K$ and $M$ be the number of users and RRHs in the macrocell respectively. Denote $\alpha, \beta_{m}, a_{k}$ and $b_{m, k}$ the following binary parameters:

$$
\begin{aligned}
& \alpha=\left\{\begin{array}{l}
1 \text { if the } \mathrm{M}-\mathrm{RRH} \text { is active } \\
0 \text { otherwise }
\end{array}\right. \\
& \beta_{m}=\left\{\begin{array}{l}
1 \text { if the RRH } m \text { is active; } m=1, \ldots, M \\
0 \text { otherwise }
\end{array}\right.
\end{aligned}
$$

$a_{k}=\left\{\begin{array}{l}1 \text { if the user } k \text { is served by the M }-\mathrm{RRH} ; k=1, \ldots, K \\ 0 \text { otherwise }\end{array}\right.$

$b_{m, k}=\left\{\begin{array}{l}1 \text { if the user } k \text { is served by the RRH } m ; k=1, \ldots, K \\ 0 \text { otherwise }\end{array}\right.$

The total consumed power $P_{M-R R H}$ at the M-RRH fronthaul is then expressed as follows:

$$
P_{M-R R H}=\alpha\left(P_{c c, M-R R H}+\sum_{k=1}^{K} a_{k} p_{k}\right)+(1-\alpha) P_{M-R R H, \text { idle }}
$$

where:

- $\quad P_{C C, M-R R H}$ is the consumed power at the circuits of the M$\mathrm{RRH}$ when the latter is active.

- $p_{k}$ is the required transmit power from the M-RRH to the user $k$ to meet his QoS

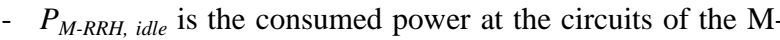
RRH when the latter is idle (i.e. not serving any user inside the macrocell)

Similarly, the total power consumed by the total RRHs inside the macrocell is given by:

$$
P_{m}=\beta_{m}\left(P_{c c, m}+\sum_{k=1}^{K} b_{m, k} p_{m, k}\right)+\left(1-\beta_{m}\right) P_{m, i d l e}
$$

where:

- $P_{C C, m}$ is the consumed power at the circuits of the RRH when the latter is active.

- $P_{m, k}$ is the required transmit power from the serving RRH to the user $k$ to meet his QoS

- $P_{m \text {, idle }}$ is the consumed power at the circuits of the RRH when the latter is idle (i.e. not serving any user around)

Consequently, the total power consumed on the fronthaul link is equal to:

$$
P=P_{M-R R H}+\sum_{m=1}^{M} P_{m}
$$

The problem formulation is therefore expressed as:

$$
\min _{\alpha, \beta_{m} a_{k}, b_{m, k}} P
$$

subject to:

$$
P_{M-R R H_{-} \text {Thres }} \leq \sum_{k=1}^{K} a_{k} p_{k} \leq P_{M-R R H_{-} \text {max }} \text { if } \alpha=1
$$

and

$$
P_{R R H_{-} \text {Thres }} \leq \sum_{k=1}^{K} b_{m, k} p_{m, k} \leq P_{R R H_{-} \text {max }} \text { if } \beta_{m}=1
$$

where:

- $P_{M-R R H_{-} \text {Thres }}$ and $P_{R R H_{-} \text {Thres }}$ are certain total transmit power thresholds at the M-RRH and the different RRH respectively.

- $P_{M-R R H \_ \text {max }}$ and $P_{R R H \_ \text {max }}$ are the maximum transmit power at the M-RRH and the different RRH respectively.

\section{PROPOSED ENERGY-BASED RRH ASSIGNEMENT ALGORITHM}

The proposed Energy-based RRH assignment scheme is a two-step algorithm. In the first step, a typical RRH assignment is applied where users are attached to the RRH presenting the lowest transmit power enabling them to meet their required QoS. In this step, all RRHs (including the M$\mathrm{RRH}$ ) are thus assumed to be active and serve the users with the lowest possible transmit power to ensure users' quality of 
service. This phase corresponds to a common attachment scheme. However, it is not optimal in terms of energy consumption as some of the RRH (including the M-RRH) keep consuming circuit power even if the number of the attached users is small. The second step is thus introduced to take advantage of the H-CRAN architecture and better coordinate between the different RRH to optimize the energy consumption in the whole fronthaul link.

The second step basic idea is to switch users from the RRHs who are underutilized to more loaded RRHs while ensuring the quality of service to these users and guaranteeing that the total consumed power in the network is minimized.

More precisely, in the second step, first the RRHs (including $\mathrm{M}-\mathrm{RRH}$ ) whose the total consumed powers are under a certain threshold $\mathrm{P}_{\text {RRH_Thresh }}$ (or $P_{M-R R H \_T h r e s h}$ ) are switched off. Then, the attached users of these RRHs are redirected to other RRHs according to the following: for each user, the active RRHs' transmit powers that ensure meet the required quality of service are sorted. Then, the user is assigned to the RRH that presents the lowest transmit power if this reassignment does not cause any exceed in the total transmit power of the RRH nor in the total consumed power in the network, otherwise, the user is assigned to the following ranked RRH verifying this condition. The algorithm ends when all users are reassigned to different RRHs. In order to ensure the feasibility of the algorithm, it is assumed that the whole network is not overloaded to be able to reassign all users. Indeed, the proposed algorithm only concerns networks which are not overloaded to save energy in those networks. In the opposite case (for networks overloaded), it is obvious that all RRHs should be activated to serve all users' demands.

Table I gives more details about the proposed energy-based RRH assignment scheme.

\section{SIMULATION RESULTS}

In this section, the performance of the proposed energy-based $\mathrm{RRH}$ assignment is evaluated in terms of total consumed power in the fronthall and compared these performances to those obtained with the common RRH assignment scheme which corresponds to the Step one of the algorithm. The impact of the number of users in the network which represents the system load, the number of the RRHs (or small cells) in the network and the consumed power threshold $P_{\text {Thres }}$ is also examined.

The proposed scheme will be referred as E-RRH Assignment (Energy-based RRH) whereas the common scheme will be called C-RRH Assignment (Common RRH Assignment).

On the other hand, two scenarios are considered. In the first scenario, a uniform distribution of all users in the network is assumed, whereas in the second scenario, the users are gathered in hotspots. For a better illustration of the second scenario, the users are randomly distributed in one area of the macrocell. Fig. 2 illustrates an example of snapshots obtained in each scenario. The simulation results given hereafter are obtained by averaging the resulting performance metrics of 1000 snapshots. Some simulation parameters are details in Table II. Note that for a better illustration of the proposed Energy-based RRH assignment scheme, the powers $P_{M-R R H \text {,idle }}$ and $P_{m, \text { idle }}$ for all $m$ are set to zero.
Table I. Proposed Energy-based RRH assignment scheme

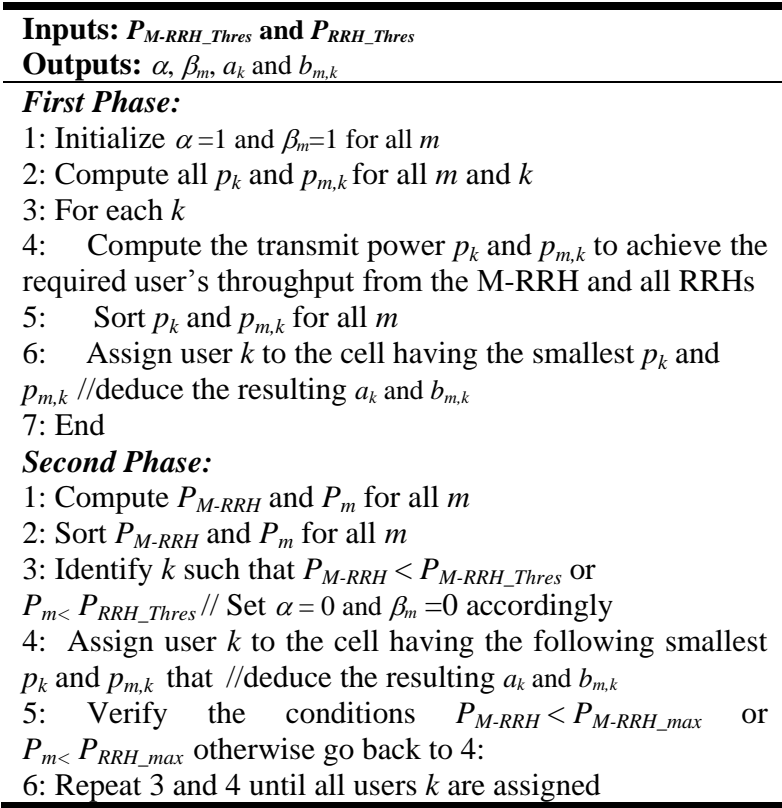

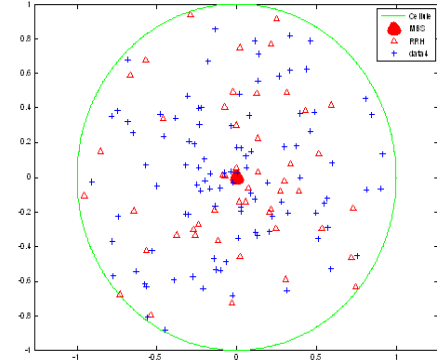

(a)

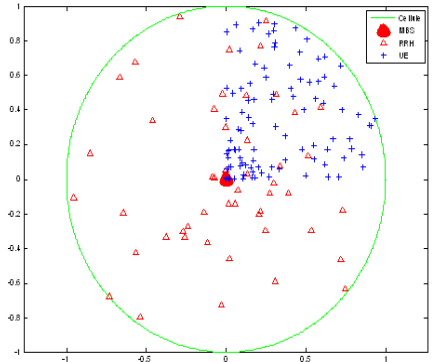

(b)
Fig. 2: (a) Scenario 1 with uniform user's distribution; (b) Scenario 2 with users distributed in a hotspot

Table II. Simulation Parameters

\begin{tabular}{|l|l|}
\hline \multicolumn{1}{|c|}{ Parameter } & \multicolumn{1}{c|}{ Value } \\
\hline Maximum transmit power at the M-RRH & 20 \\
$P_{M-R R H_{\text {max }}}[\mathrm{W}]$ & \\
\hline $\begin{array}{l}\text { Maximum transmit power at the M-RRH } \\
P_{R H_{\_} \max }[\mathrm{mW}]\end{array}$ & 200 \\
\hline $\begin{array}{l}\text { Consumed power at the circuits of the M- } \\
\text { RRH } P_{C C, M-R R H}[\mathrm{~W}]\end{array}$ & 4 \\
\hline $\begin{array}{l}\text { Consumed power at the circuits of the RRH } \\
P_{C C, m}[\mathrm{~mW}]\end{array}$ & 40 \\
\hline $\begin{array}{l}\text { Minimum required users' throughput } \\
{[\text { Mbps] }}\end{array}$ & 2 \\
\hline Macrocell radius [km] & 0.5 \\
\hline Noise power density & $-174 \mathrm{dBM} / \mathrm{Hz}$ \\
\hline Pathloss between a SU Tx and a SU Rx & $130.62+37.6 \log _{10}\left(d_{s s}\right)$ \\
\hline
\end{tabular}

\subsection{Impact of the system load}

Fig. 3 depicts the total consumed power vs. the number of users in the macrocell $K$ for both scenarios with $P_{M}$ RRH_Thres $=0.5 P_{M-R R H_{-} \max }, P_{R R H_{-} \text {Thres }}=0.5 P_{R R H_{\_} \max }$ and $M=50$. It shows that in both scenarios, the power consumed increases with the number of UEs. However, the classical C-RRH Assignment scheme in scenario 1 consumes more power as compared to the scenario 2 . In the first case this power varies from $6 \mathrm{~W}$ (15 UE) to $7.1 \mathrm{~W}$ (305UE). As for the proposed E- 
RRH Assignment scheme, the total consumed power $P$ in the system in the first scenario varies from $0.68 \mathrm{~W}$ (15UEs) to $2.91 \mathrm{~W}$ (305 UEs). In scenario 2 this power increases from $0.37 \mathrm{~W}$ (15UEs) to $1.42 \mathrm{~W}$ (305) W.

This corresponds to a reduction in power consumption from 88.66\% (15UEs) to 58.95\% (305 EUs) for the scenario1 against $93.8 \%$ (15 EUs) to $77.24 \%$ for the scenario2 as shown in Fig. 4. In both scenarios, the proposed E-RRH Assignment scheme considerably reduces the consumed power in the system. This reduction is even more important in the scenario 2 where the UEs are concentrated in one area of the macrocell. In fact, when the UEs are concentrated in one zone of the cell, the primary and secondary choices of the UEs are the RRHs present in this zone. RRHs not in this area would transmit higher power and thus be less likely to be assigned to a UE; they will as a consequence be deactivated. The number of RRHs put on standby is higher in this case. The percentages of RRHs extinguished in scenario 2 confirms this, with a rate of extinguished RRHs decreasing from $93 \%$ to $83 \%$, as compared to a rate that decreases from $88 \%$ to $68 \%$ in the scenario 1 (Fig. 5).

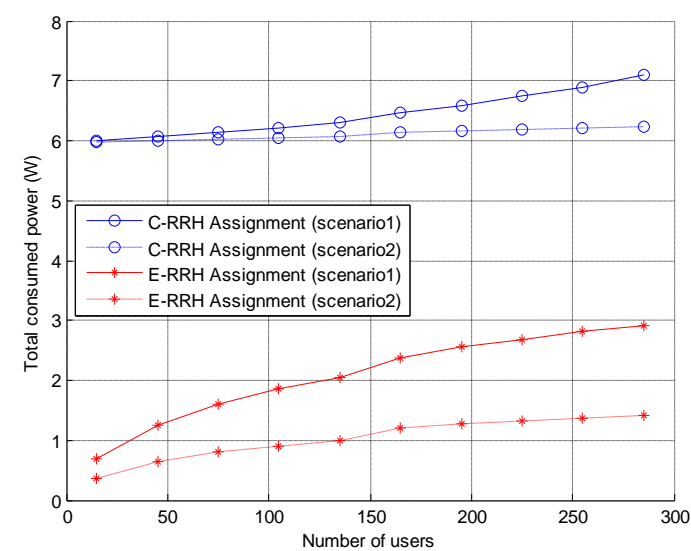

Fig. 3: Total consumed power in the system vs. the number of users for $\mathbf{P}_{\mathrm{M} \text {-RRH_Thres }}=\mathbf{0 . 5} \mathbf{P}_{\text {M-RRH_max }}$, $\mathbf{P}_{\text {RRH_Thres }}=0.5 \mathbf{P}_{\text {RRH_max }}$ and $\mathrm{M}=\mathbf{5 0}$.

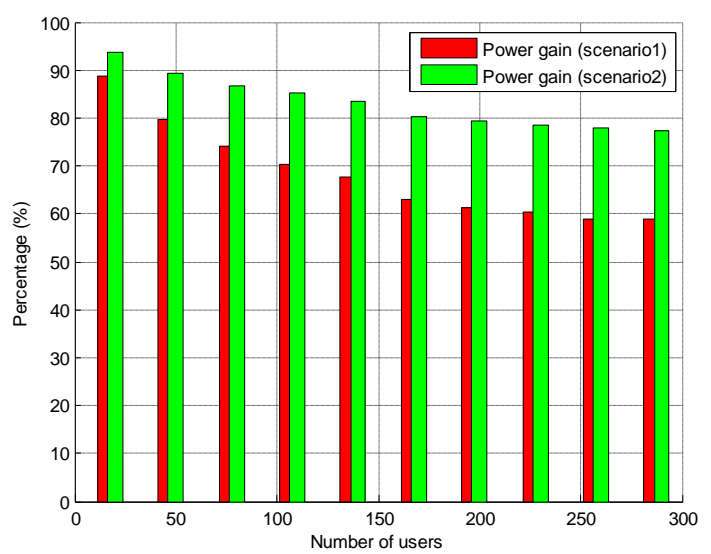

Fig. 4: Percentage of the power gain for the proposed E-RRH Assignment scheme vs. the number of users.

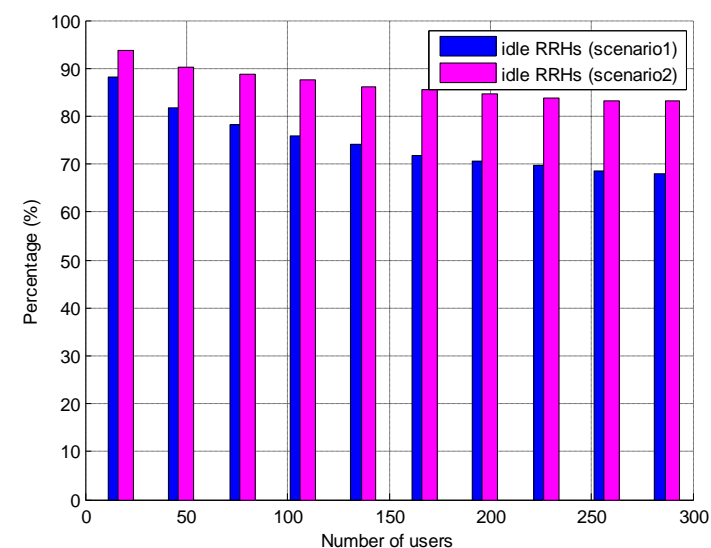

Fig. 5: Percentage of the idle RRHs for the proposed E-RRH Assignment scheme vs. the number of users.

\subsection{Impact of the number of RRHs}

Fig. 6 depicts the total consumed power vs. the number of RRHs in the macrocell $M$ for both scenarios with a total number of users of $K=150$ and $P_{M-R R H_{-} \text {Thres }}=0.5 P_{M-R R H_{-} \max }$, $P_{R R H \_ \text {Thres }}=0.5 P_{R R H \_m a x}$. The analysis of the two scenarios shows us that when there are fewer resources that is to say less than RRHs in the cell, the power consumed is high. In the first scenario, for both RRH assignment schemes $P=24.46 \mathrm{~W}$ when there are 5 RRHs in the network. In scenario 2, the total consumed power for both RRH assignment schemes is equal to $P=24 \mathrm{~W}$. This means that regardless of the distribution of UEs in the cell, when there is less resource the powers transmitter, the total consumed power is almost identical for both assignment schemes. In fact, when there are fewer RRHs all resources are used, no RRH should be put on standby.

However, as the number of RRHs increases, the power consumed begins to fall sharply. In scenario 1 the power drops from $24.46 \mathrm{~W}$ to $15 \mathrm{~W}$ when the number of RRHs goes from 5 to 15 for the conventional C-RRH assignment scheme. As for the proposed E-RRH Assignment scheme, the power drops from $24.46 \mathrm{~W}$ to $9.93 \mathrm{~W}$ for the same variation in the number of RRHs. This corresponds to a reduction of $34 \%$ of the consumed power. When the number of RRHs reaches 25 , the total consumed power drops to $2.26 \mathrm{~W}$ which implies a reduction of $72 \%$ thanks to the proposed E-RRH assignment scheme as compared to the conventional C-RRH assignment scheme. Beyond $25 \mathrm{RRHs}$, there is a total consumed power reduction between $65 \%$ and $74 \%$. The total power consumed by the proposed E-RRH assignment scheme varies between $2 \mathrm{~W}$ and $2.36 \mathrm{~W}$ (Fig. 7). For the scenario 2, when the number of RRHs increases from 5 to 15 RRHs, the total consumed power drops from $24 \mathrm{~W}$ to $1.47 \mathrm{~W}$. This corresponds to a reduction of about $93 \%$. From $25 \mathrm{RRHs}$ the power consumed by the proposed E-RRH assignment scheme varies between $1.16 \mathrm{~W}$ and $1.65 \mathrm{~W}$. This corresponds to a reduction in consumed power that varies between $75.86 \%$ and $82.81 \%$.

On the other hand, the proposed E-RRH assignment scheme significantly reduces the power consumed when the number of RRHs increases. This is due to the fact that with the increase of power transmitters (i.e. the RRHs), a high number of underutilized RRHs for the same system load (i.e. the same number of users in the system) is obtained. It is thus preferable to switch these inactive RRH using the proposed ERRH assignment scheme. 
Furthermore, as shown in Fig. 8, this reduction is higher in scenario 2, because when the number of RRHs increases, the concentration of the number of RRHs also increases in the hotspot where the UEs are concentrated. They are therefore closer to their first choices and their secondary choices. This reduces the power required at the RRHs in this area to meet their quality of service (i.e. the required throughputs). Moreover, this transmit power corresponds practically to the power consumed by the entire network. The other RRHs are also too far from the UE concentration area.

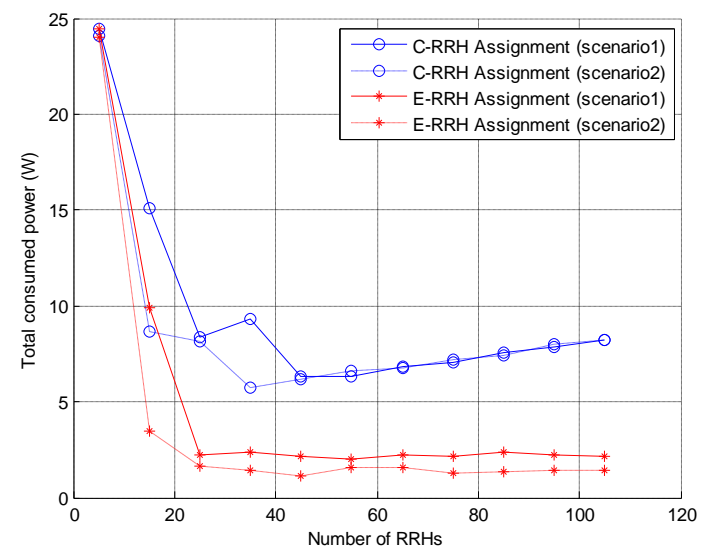

Fig. 6: Total consumed power in the system vs. the number of RRHs for $\mathbf{P}_{\mathrm{M}-\mathrm{RRH} \text {-Thres }}=0.5 \mathbf{P}_{\mathrm{M}-\mathrm{RRH} \text { max }}$, $P_{\text {RRH_Thres }}=0.5 P_{\text {RRH_max }}$ and $K=150$.

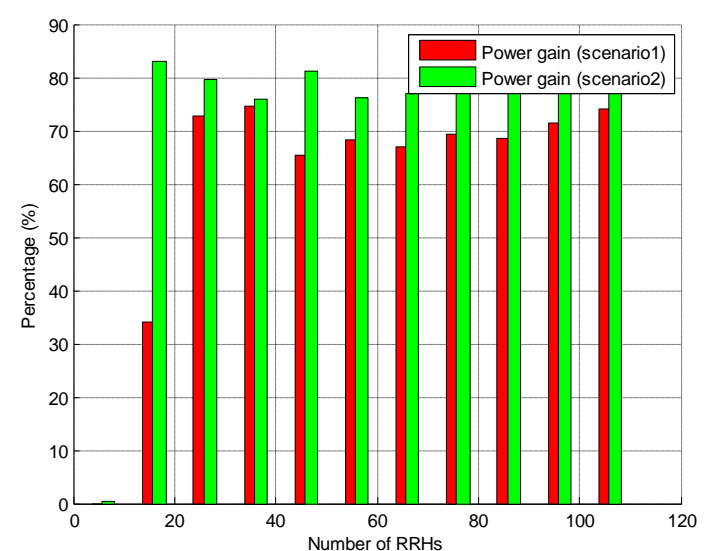

Fig. 7: Percentage of the power gain for the proposed ERRH Assignment scheme vs. the number of RRHs.

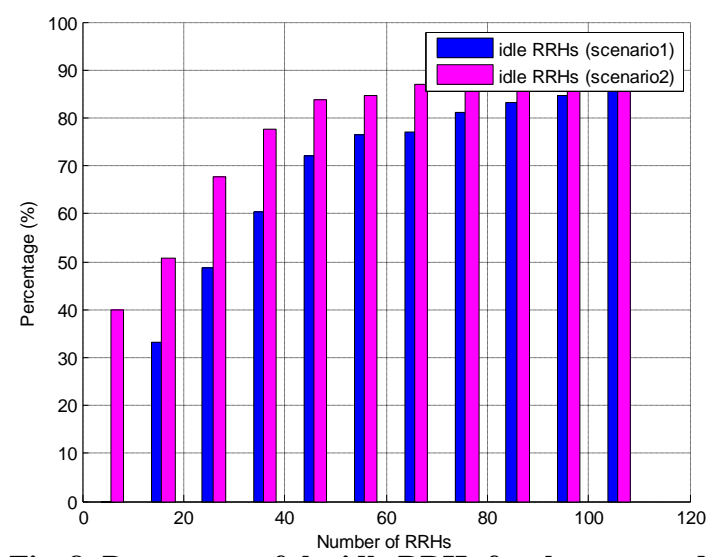

Fig. 8: Percentage of the idle RRHs for the proposed E-RRH Assignment scheme vs. the RRHs.

\subsection{Impact of the consumed power threshold}

Fig. 9 depicts the total consumed power vs. the threshold radio (i.e. which corresponds to is the ratio of $P_{M-R R H_{-} \max }$ to $P_{M-R R H_{-} \text {Thres }}$ ) for both scenarios with a total number of users int the macrocell of $K=150$ and a total number of RRHs equal to $M=50$. It shows that in both scenarios, the proposed E-RRH assignment scheme reduces the total consumed power in both scenarios. In both cases, the power consumed is constant regardless of the power threshold for the conventional C-RRH assignment scheme. It is equal to $6.33 \mathrm{~W}$ in the scenario 1 and to $6.1 \mathrm{~W}$ in the scéanrio2. This steadiness is due to the fact that this scheme only depends on the number of UEs and the RRHs in the network which are constant in this simulation. Each UE is normally associated with its first choice of RRHs to meet the required quality of service.

On the other hand, when the proposed E-RRH assignment scheme is applied in scenario 1 where the UEs are uniformly distributed throughout the cell, there is a notable reduction in the power consumption. This power increases by $1.63 \mathrm{~W}$ (threshold ratio $=0.1$ ) to reach $2.13 \mathrm{~W}$ (threshold ratio $=0.5 \mathrm{~W}$ ). Then, it slightly decreases to reach $1.83 \mathrm{~W}$ (threshold ratio=0.8). The proposed E-RRH assignment scheme achieves a reduction of total consumed power between $66.37 \%$ (threshold ratio $=0.5$ ) and $74.22 \%$ (threshold ratio $=0.1$ ) as shown in Fig. 10, and switches into "sleep mode" (or idle mode) between $71.43 \%$ (threshold ratio $=0.1$ ) and $75 \%$ (threshold ratio $=03$ ) of the RRHs as shown if Fig. 11. For a lower threshold ratio, the rate of extinguished RRHs is lower, because in this case there are more RRHs whose transmit powers exceed the maximum allowed transmit power. As the threshold ratio increases, the number of RRHs extinguished increases because the number of RRHs satisfying the condition decreases. So the number of UEs redirected to their secondary choices increases. The transmit power of the former assigned RRHs increases in return. This could explain the slight increase despite the fact that the number of RRHs extinguished increases with the value of the threshold ratio.

In scenario 2, i.e. when the UEs are grouped in one area of the macrocell, one can note that with the proposed E-RRH assignment scheme, the total consumed power is lower as compared to the scenario 1 . It varies between $0.72 \mathrm{~W}$ (threshold ratio $=0.1$ ) and stabilizes around $1 \mathrm{~W}$ from a threshold ratio of 0.4 . It is also observed that the total consumed power decreases when the threshold ratio increases (from $88 \%$ to $82 \%$ ). This means that there are fewer RRHs that meet the condition, the same in the scenario 1. However, contrary to the scenario 1 , after redirection of the UEs attached to the under utilized RRHs towards their secondary choice, the total consumed power using the proposed E-RRH assignment scheme is lower. This is explained by the fact that the secondary RRHs of the UEs are closer to the UEs, contrary to the scenario 1 . So the secondary choices of the UEs transmit less power as compared to the scenario1.

From this study, it can be deduced that when the UEs are concentrated in one hotspot, the total consumed power using the proposed E-RRH assignment scheme is considerably lower than the power consumed when the conventional CRRH assignment scheme is applied. Consequently, the proposed E-RRH assignment scheme helps better coordinate the energy consumption in the network and takes better advantage of the system architecture of the H-CRAN. 


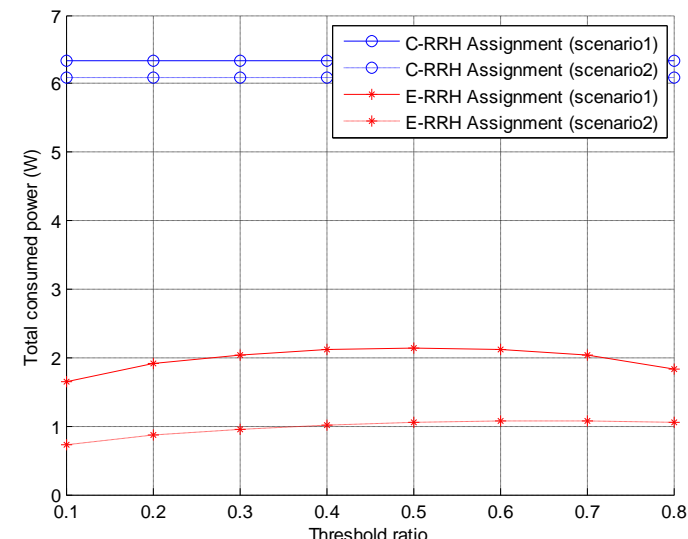

Fig. 9: Total consumed power in the system vs. the number of users for $K=150$ and $M=50$.

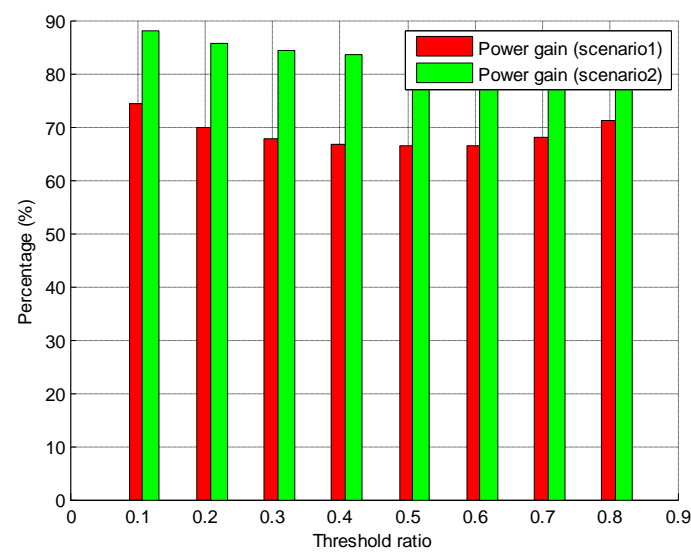

Fig. 10: Percentage of the power gain for the proposed E-RRH Assignment scheme vs. the threshold ratio $(K=150$ and $M=50)$.

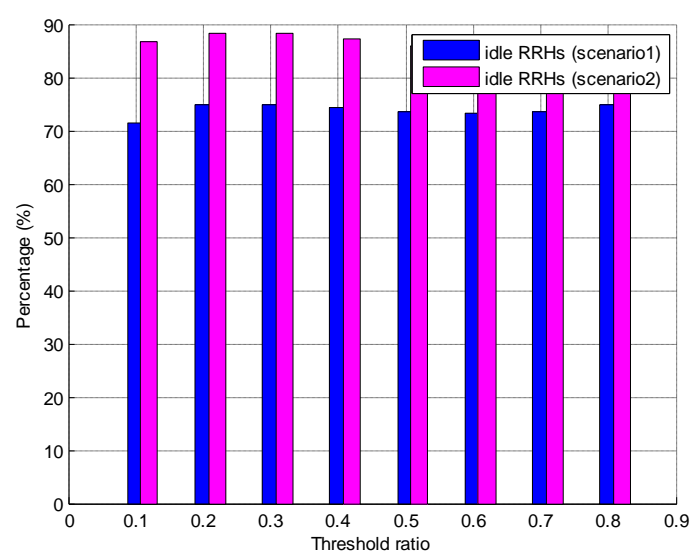

Fig. 11: Percentage of the idle RRHs for the proposed E-RRH Assignment scheme vs. the threshold ratio $(K=150$ and $M=50$ ).

\section{CONCLUSION}

The paper aimed at designing a new Energy-based RRH assignment scheme to reduce the total power consumption in a 5G H-CRAN network.

An algorithm which switches to an idle mode all RRHs whose transmit power is below a certain power threshold is defined. Then the UEs are assigned to other RRHs in the network without exceeding the total transmit power in the system and while meeting their required throughput. In this paper, the evolution of the total consumed power with a uniform distribution of UEs and distribution of the UEs in a hotspot and according to three parameters: the number of UEs in the network, the number of RRHs in the macrocell and the power threshold is analyzed.

It is shown that the proposed Energy-based RRH assignment scheme reduces the total consumed power by more than $60 \%$ when the UEs are uniformly distributed in the network and by more than $80 \%$ when the UEs are concentrated in one area of the macrocell. This power gain can increase with the increase of the cell load and the number of RRHs in the network especially when the threshold ratio is properly chosen.

Future research will look at the impact of the proposed Energy-based RRH assignment scheme on the activation of some BBUs inside the BBU pool of the H-CRAN in order to further improve the Energy Efficiency of the system.

\section{REFERENCES}

[1] PENG, Mugen, YU, Yuling, XIANG, Hongyu. Energyefficient resource allocation optimization for multimedia heterogeneous cloud radio access networks. IEEE transactions on Multimedia, 2016, vol. 18, no 5, p. 879892.

[2] PENG, Mugen, LI, Yuan, JIANG, Jiamo. Heterogeneous cloud radio access networks: A new perspective for enhancing spectral and energy efficiencies. IEEE Wireless Communications, 2014, vol. 21, no 6, p. 126135 .

[3] PENG, Mugen, LI, Yong, ZHAO, Zhongyuan. System architecture and key technologies for $5 \mathrm{G}$ heterogeneous cloud radio access networks. IEEE network, 2015, vol. 29, no 2, p. 6-14.

[4] KULLIN, C, RAN, D. C-ran the road towards green ran. China Mobile Research Institute, White Paper, 2011

[5] NAMBA, Shinobu, WARABINO, Takayuki, KANEKO, Shoji. BBU-RRH switching schemes for centralized RAN. In : Communications and Networking in China (CHINACOM), 2012 7th International ICST Conference on. IEEE, 2012. p. 762-766.

[6] BOULOS, Karen, EL HELOU, Melhem, LAHOUD, Samer. RRH clustering in cloud radio access networks. In: Applied Research in Computer Science and Engineering (ICAR), 2015 International Conference on. IEEE, 2015. p. 1-6.

[7] XU, S., WANG, S. (2016, May). Efficient Algorithm for Baseband Unit Pool Planning in Cloud Radio Access Networks. In Vehicular Technology Conference (VTC Spring), 2016 IEEE 83rd (pp. 1-5). IEEE.

[8] LEE, Ying Loong, WANG, Li-Chun, CHUAH, Teong Chee. Joint Resource Allocation and User Association for Heterogeneous Cloud Radio Access Networks. In : Teletraffic Congress (ITC 28), 2016 28th International. IEEE, 2016. p. 87-93.

[9] DOLFI, Marco, MOROSI, Simone, CAVDAR, Cicek. Energy efficient optimization of a sleep mode strategy in heterogeneous cellular networks. In : Networks and Communications (EuCNC), 2017 European Conference on. IEEE, 2017. p. 1-6. 
[10] ZUO, Jun, ZHANG, Jun, YUEN, Chau. Energy efficient user association for cloud radio access networks. IEEE Access, 2016, vol. 4, p. 2429-2438.

[11] DAI, Yongyu, JIN, Shi, PAN, Leyuan. Interference control based on beamforming coordination for heterogeneous network with RRH deployment. IEEE Systems Journal, 2015, vol. 9, no 1, p. 58-64.
[12] KHAN, M., ALHUMAIMA, RAAD S., ALRAWESHIDY, HAMED S. Reducing energy consumption by dynamic resource allocation in C-RAN In: Networks and Communications (EuCNC), 2015 European Conference on. IEEE, 2015. p. 169-174. 